\title{
Transformando la educación para la paz en cultura de paz por medio de la eticidad y el pensamiento crítico
}

\author{
Transforming education for peace into a culture \\ of peace through ethics and critical thought
}

\author{
Juan Alanis Aguilar \\ Universidad Autónoma del Estado de Morelos \\ https://orcid.org/0000-0003-3467-5228 \\ alanisaguilar.juan@gmail.com
}

Fecha de recepción: 31/10/2021

Fecha de aceptación: 09/12/2021

\section{Resumen}

En el siguiente artículo exploraremos algunas definiciones de paz que se han construido después de la Segunda Guerra Mundial, así como las posturas que han tenido algunos autores respecto a este tema. Seguiremos con la explicación del pensamiento crítico, por medio de diversas descripciones y posturas teóricas, así como la construcción de un sujeto crítico, pues se considera a este, por lo menos en este trabajo, como una pieza fundamental para construir una cultura de paz, a través de la educación para la paz. Posteriormente, encaminaremos los esfuerzos en vincular estos dos aspectos con otros dos más: la eticidad y la formación. Sin ellos, según nuestro entender, cualquier ejercicio para transformar la cultura por el hecho de cambiarla sería fútil, ya que se trata de un proceso gradual que dispone de muchas habilidades y capacidades de los sujetos para su realización, además de un ejercicio práxico, que requiere de quien lo lleva a cabo un alto grado de responsabilidad consigo mismo y para con las otredades.

Palabras clave: paz, pensamiento crítico, educación para la paz, cultura de paz, eticidad. 


\section{Abstract}

This article begins by exploring some influential definitions of peace that have been constructed since the Second World War. It first reviews the postures that certain authors have adopted regarding the question of peace, followed by an elucidation of critical thought that takes the reader through various descriptions and theoretical positions while also addressing the construction of the critical subject. The study considers the critical subject to be a fundamental piece in the construction of a culture of peace achieved through education for peace. We then connect two additional aspects to assemble an apparatus with four axes of operation. These two elements are ethics and training. Without them, any exercise that seeks to transform culture would be futile, because this is a gradual process that requires many abilities and capacities of the subjects involved for its realization, together with exercises of praxis that demand a high degree of responsibility-with both themselves and others- on the part of the individuals who participate.

Keywords: peace, critical thought, education for peace, culture of peace, ethics.

\section{Introducción}

Durante los últimos años, el tema de la paz ha estado presente en políticas públicas, instituciones educativas, centros de trabajo e incluso ocasionalmente en nuestras charlas cotidianas. Esto ha ocurrido ya sea para ir ganando seguidores o curiosos sobre el tema; o bien para buscar un discurso que llene de esperanza el camino hacia una transformación social tan requerida y deseada, pero muy pocas veces buscada en su ejecución.

Esta transformación social no es fácil. Cuando construimos una cultura de paz, desde una educación para la paz, nos enfrentamos a siglos de una cultura caracterizados por invisibilizar, por prejuicios y ejercicios irreflexivos, o bien a perspectivas de la paz inflexibles que muchas veces se contraponen a lo que aquí se puede entender por este concepto. En otras ocasiones, se quiere que la paz llegue por sí misma, sin esfuerzo ni compromiso alguno de los individuos, simplemente que surja y se quede, estática, inamovible, eterna; sin embargo, como iremos revisando a lo largo de este trabajo, construir una cultura de paz desde una educación para la paz es un proceso largo, que requiere de voluntad, atención y responsabilidad de quienes estén dispuestos a emprender dicho camino.

Lo anterior se debe, en gran medida, a que la cultura de la violencia no ha tenido un freno ni ha sido expuesta durante siglos, por el contrario, se ha normalizado; incluso aquellos que hablan desde el privilegio o una posición de poder, 
la defienden. La cultura de la violencia no requiere de esfuerzo, capacitación, responsabilidad, disposición ni atención, basta con reproducir lo visto y aprendido sin crítica y de manera mecánica.

Además de lo ya mencionado, al construir paz, se presentan diferentes vertientes, las cuales nos ofrecen alternativas que se ajustan a diversos niveles de realidad, de contextos y de violencias. Con ello, a su vez, se compone un concepto de paz para las particularidades requeridas; sin embargo, la utilidad de este se extiende al unificarlo, convirtiéndolo en complejo y extenso sin limitarse a algunas acepciones, como lo económico, social, cognitivo, emocional, asimismo la justicia, la libertad, sin olvidar la responsabilidad, la resiliencia, la empatía, entre otros.

$\mathrm{Al}$ abarcar tanto, la paz y su concepto deben mediarse con ejercicios reflexivos; para ello, el pensamiento crítico resulta uno de los filtros indispensables al construir y educar para la paz. Esto se debe a que dicha construcción, aparentemente teórica, también busca construir y transformar a largo plazo la realidad y la cultura en la que se desarrollan los sujetos con quienes la llevamos a la acción. En este sentido, dicho proceso se vuelve consciente y emancipador, por no sólo hablar de paz, sino al educar en paz para su propia transformación, así como al autoevaluarse constantemente para superar y adecuarse a sí mismo dentro de la realidad donde se lleva a cabo.

Finalmente, cabe decir que educar para la paz es accionar para que las brechas se puedan disipar y dar dignidad al ser humano, emancipándolo de la violencia, de la barbarie y de una vida irreflexiva.

\section{Educación para la paz}

Cuando se habla de cultura o educación para la paz, muchas personas imaginan un mundo sin guerras, un lugar lejano sin conflictos ni confrontaciones; lleno de utopías y personas vestidas de blanco, en un ambiente celestial, como en el que cayó el filósofo Tales de Mileto en el diálogo donde Platón de Teeteto (2017) nos habla sobre la naturaleza del saber. Asimismo, existen enfoques que apenas si se relacionan al tema, que no están fundamentados de manera rigurosa y que presentan la paz como una alternativa espiritual o metafísica en la que, por mero trabajo personal interiorizado, va a modificarse todo el contexto social, violento y criminal que existe; y aunque sí se busca una interiorización del tema, también es necesaria la acción social que viene junto con ella, como un ejercicio disciplinado y autodirigido que busque la justicia social y el bienestar en las comunidades.

Esos enfoques mencionados anteriormente son los que hacen que la labor de educar para la paz y de construir una cultura de paz se tornen en un ejercicio utópico, y se entienda como fraude o una pérdida de tiempo, así como un ideal 
espiritual más bien sectario o cargado de alguna religión. Pero esto no es paz, por lo menos no desde las posturas teóricas que a continuación se estarán enmarcando. Lo primero que hay que mencionar es que la educación para la paz, por lo menos en México, lleva más de 30 años desarrollándose desde múltiples visiones teóricas rigurosas, por lo que mencionaremos a algunos de los autores fundamentales y cimentaremos la visión que se está teniendo de paz vinculada al pensamiento crítico.

En las siguientes líneas veremos cómo el concepto de paz es más complejo de lo que creemos, pues es un ejercicio práxico; esto quiere decir que se requiere entender la parte teórica y, a su vez, llevarla al campo de acción. En este sentido, Adolfo Sánchez Vázquez (1969) menciona en su filosofía que estas prácticas deben ser praxiológicas y no ontológicas, o lo que es lo mismo, no puede haber juicio sobre un problema ontológico, antropológico y epistemológico al margen de la praxis (Alanis, 2015). Por esto, la educación para la paz tiene tanta relevancia, pues se convierte en un ejercicio praxiológico donde además de tener fundamentos de la teoría, ejercitamos habilidades, generamos juicios de valor sobre acciones realizadas, buscando un bien común y preferentemente una justicia social.

Para comenzar, respondiendo a los principios de la teología negativa o vía negativa, ${ }^{1}$ Galtung distingue que para hablar de una construcción de paz debemos entender todo aquello que no es paz, es decir, todo aquello que es violencia. En este sentido, el entendimiento de la violencia se ha vuelto fundamental para retomar acciones que la contrarresten (Galtung, 2003).

Entonces, lo más aceptado en términos generales es la definición de violencia que propone la Organización Mundial de la Salud (OMS). En ella se entiende por violencia el uso intencional de la fuerza o el poder físico, de hecho o como amenaza, contra uno mismo, otra persona, o un grupo de comunidad, que cause o tenga muchas probabilidades de causar lesiones, muerte, daños psicológicos, trastornos del desarrollo o privaciones. Ahora bien, el texto de la OMS data del 2002, por lo que hoy en día esta definición puede quedarse un poco corta ante las nuevas problemáticas sociales y variables que han surgido gracias a investigaciones académicas y rigurosas de lo que es la violencia y sus tipos.

De modo que iniciaré hablando sobre las investigaciones que Johan Galtung realizó en los años sesenta, ya que categoriza la violencia como no se había hecho hasta ese momento, y la contrapuntea con la construcción de paz desde el conflicto. En ese entonces, muy pocos, sino más bien ningún autor anterior, se habían detenido a entender y explicar que la violencia tiene múltiples acepciones, lo cual se convirtió en punta de lanza para las investigaciones posteriores sobre paz. También, por esa época él mismo funda el Instituto de Investigación para la Paz de Oslo, donde se construye el conocido triángulo de la violencia, que se

\footnotetext{
${ }^{1}$ Para esta vía, dios es incognoscible e incomprensible, solo podemos decir de dios lo que no es, pues todo lo .que es, lo que conocemos y comprendemos no es divino y además es finito.
} 
retoma del texto Tras la violencia, 3R: reconstrucción, reconciliación, resolución. Afrontando los efectos visibles e invisibles de la guerra y la violencia (2003). A partir del cual se dividen tres aspectos de la violencia interrelacionados, pero a su vez separados por una taxonomía de visibilidad; esto quiere decir que existen violencias más manifiestas y observables que otras, donde el punto en común siempre es el abuso de poder (Galtung, 2003); estos tres tipos de violencia son:

- Violencia Directa: se ejerce entre personas entre las cuales puede haber relaciones asimétricas de poder; se practica de manera directa y visible; puede ser física, verbal o psicológica; acciones que atentan en contra de la integridad del otro.

- Violencia Cultural: las manifestaciones que perpetúan la idea del abuso, la naturalización de estereotipos y de relaciones inequitativas de poder.

- Violencia Estructural: entendida como la distribución desigual de recursos, ingresos y riqueza; tiene que ver con las formas en las que la estructura permite ese abuso de poder desde el sistema económico-político.

Una vez que logramos categorizar bajo esta taxonomía de Galtung los tipos de violencia, comenzamos a observar con nuevos lentes las interacciones entre individuos, sobre todo por las violencias que se mantienen invisibles a los ojos del observador que no está habituado a los conceptos y sus formas. No obstante, para los ojos entrenados de quienes construyen paz, comienza a ser muy obvio identificar muchas de las manifestaciones presentes en el día a día, pero aun con todo requerimos un análisis más profundo de cada una de las acciones, interacciones, manifestaciones y su contrapunto.

Una vez que tenemos sobre la mesa la importancia que tiene el entender la violencia en las investigaciones de paz, podemos hacer un rápido recorrido a las concepciones que se han construido de esta última. Muchas de estas definiciones existen por la relación entre la guerra ${ }^{2}$ y la paz, en una dicotomía o, mejor, en un continuum (De Vera, 2016), donde si existe uno, el otro no está presente y viceversa, se establece una relación de oposición excluyente. Para hacer clara dicha relación entre guerra y paz, a continuación, se comparten algunas definiciones y autores que hablan de la paz desde un enfoque filosófico:

- La pax romana: se habla de un trato igualitario en condiciones para acordar, es un pacto verbal, un trato, un convenio. Ausencia de conflictos bélicos (Jares, 2001).

- Sócrates: estado de participación donde las ideas sean superiores a los intereses personales por medio de un ejercicio dialógico mayéutico (Platón, 2014).

- Aristóteles: aunque nunca escribió un tratado sobre la paz, podemos encontrar una importante elaboración de aquellos elementos necesarios que

\footnotetext{
${ }^{2}$ En este trabajo no definiremos lo que es la guerra.
} 
contribuyen a crear un estado de paz civil, tal como la necesidad de promover la amistad, el diálogo, la democracia, el estado de derecho, la ley y la felicidad. (Bosé y Fernández, 2017).

- René Descartes: no menciona directamente la construcción de paz, pero sí un ejercicio de conciencia del ser humano en el mundo, pues no sólo se requiere de voluntad, sino de ejercicio intelectivo a conciencia para aprender a manifestar juicios verdaderos acerca de las cosas del mundo (Descartes, 2011).

- Immanuel Kant: escribe La paz perpetua, donde busca el bienestar entre las naciones por medio de diversas acciones - lo que algunos pueden considerar un precedente a la ONU-, los individuos se convierten en ciudadanos del mundo, replicando acuerdos, pactos, tratos y convenios a nivel personal y social, garantizando así una convivencia pacífica que requiere la voluntad y participación de sus integrantes. Es un ejercicio intelectivo más allá de intereses particulares, generando corresponsabilidades (Kant, 2014).

Después de estos ejemplos de paz, retomaremos el concepto como objeto de estudio científico después de la Segunda Guerra Mundial. Como menciona Muñoz (2001), el horror de la guerra debía ser explicado y también relacionado con un horizonte de esperanza, lo que nos hace encontrarnos de nuevo con esta idea dicotómica.

Es también en esta 'beta de paz' que vio la luz la pedagogía de la paz como estudio de los comportamientos agresivos y violentos en los seres humanos, que apuesta por nuevas formas de socialización y educación para formar personas más libres, responsables y creativas (Muñoz, 2001). Como se verá en el apartado final, se requiere considerar a la educación como parte fundamental de la socialización humana, pues a través de ella adquirimos valores, herramientas y una visión hacia el mundo, así como una actitud más democrática frente a las injusticias.

Es en este marco, cuando comienza a desplegarse con mayor fuerza e intensidad la Investigación para la Paz como campo de estudio con identidad propia, que surgieron dos términos importantísimos: la paz positiva y la paz negativa. Los primeros acercamientos a la paz como objeto de estudio científico aportaron reflexiones interesantes que trajeron la comprensión de la paz negativa "como ausencia de guerra o como situación de no-guerra" (Muñoz, 2001); en otras palabras, cuando se construye paz a partir de la guerra se le atribuye el concepto de paz negativa, es decir, antitética a la guerra, su oxímoron incluso, como generalmente se le había atribuido con anterioridad. En segundo lugar, la paz positiva surge como una de las perspectivas que ayudaron a pensar la paz de una manera más integral, entendida como "el resultado de una construcción consciente de una paz basada en la justicia, generadora de valores positivos y perdurables, capaz de integrar política y socialmente, de generar expectativas, y de contemplar la satisfacción de las necesidades humanas” (Muñoz, 2001, p. 6). 
De manera circular en este artículo, regresamos a la idea de los primeros estudios de la investigación para la paz, los cuales se orientaban a entender la guerra y la violencia con la premisa de que si las entendemos, las podemos contrarrestar. Entre los personajes que sostienen tal postura se encuentra Smith (1979), mencionando que, paralelamente a la comprensión de la paz, hay que entender la violencia y los conflictos en los que se enmarca y adquiere su sentido (Islas, 2019), pues, como señala Martín (2003), es importante no perder de vista que "una cosa es 'ver' la violencia y plantearse qué hacer con ella, desde la violencia, y otra, muy diferente, es 'ver' violencia y 'ver' paz, y plantearse ‘qué hacer con la violencia desde la paz' (Islas, 2019).

Bajo este planteamiento, la paz positiva acciona desde sus propias herramientas y medios, como concepto ampliado, más allá del continuum de la guerra o de la violencia, y es ya un proceso complejo, se comienza a relacionar con los derechos humanos, el desarrollo humano y social, así como el respeto del medio ambiente, logrando así armonía social, justicia, igualdad y la eliminación total de la violencia estructural (Islas, 2019). Es decir, es un proceso holístico.

Por su parte, Muñoz (2001) introduce el término de paz imperfecta, al considerar que esta es inherentemente humana. Describe que una de las mayores ventajas es que puede ser sentida, percibida y pensada desde múltiples puntos, espacios y ámbitos. Sin embargo, por esa misma razón, se le ha despojado de las herramientas de la ciencia, las cuales se reservan para el estudio y el entendimiento de la violencia, dejando la paz a la sombra de cierta moral, de ciertos chamanes, charlatanes y otros individuos que lo último que querrían construir es paz. Ciertamente, que sea una paz imperfecta nos permite considerarla como inacabada, es decir, como un proceso que necesita de continuidad teórica y práctica; actitud que permitirá darle el peso y la atención justa desde la ciencia y desde la acción.

Otro término que se deriva de investigaciones más contemporáneas es el de la paz neutra de Jiménez (2011). El autor la define como una implicación activa y personal para reducir la violencia cultural y/o simbólica sobre los principios de libertad, igualdad, justicia social y responsabilidad. Esta es una propuesta de un nuevo paradigma que constituye una alternativa metodológica y conceptual para la transformación de los conflictos, pretendiendo que por vías pacíficas se eliminen o disminuyan las diversas prácticas de violencia cultural o simbólica. Lo cual nos hace recordar, una vez más, los aportes de Galtung.

Pasando a otro aspecto estrechamente relacionado con lo que se ha mencionado en los últimos párrafos, Cascón (2001) nos habla de la importancia del conflicto en la construcción de paz, pues existe la tendencia a considerar como sinónimos a "conflicto" y "violencia”. Dicho autor estima este fenómeno como una confusión, ya que toda expresión de violencia se considera un conflicto, mientras que la ausencia de violencia se considera una situación sin conflicto e incluso de paz; sin embargo, una situación se define como conflicto, no por su apariencia externa, sino por su contenido, por sus causas profundas. Como se ha mencionado, hay violencias que llegan a ser invisibles o 
invisibilizadas por el mismo sistema de creencias o coerción social, y requerimos de una visión más fina para percatarnos de tales actos.

Finalmente, e intentando integrar lo visto con los autores mencionados en este apartado, cabe aludir el trabajo de Jares, pues nos pone de manifiesto las características para concebir y aplicar la Educación para la Paz:

como un proceso educativo, dinámico, continuo y permanente, fundamentado en los conceptos de paz positiva y en la perspectiva creativa del conflicto, como elementos significantes y definidores, y que, a través de la aplicación de enfoques socioafectivos y problematizantes pretende desarrollar un nuevo tipo de cultura, la cultura de la paz, que ayude a las personas a desvelar críticamente la realidad para poder situarse ante ella y actuar en consecuencia (Jares, 1999).

La educación para la paz se convierte en un proceso complejo, completo, e integrador, no solo de una visión, sino de varias, que se congregan en un engranaje de piezas únicas, separadas, independientes, funcionales en sí mismas, que a su vez generan una sinergia que hace funcionar todo un mecanismo de manera armónica llamado cultura de paz. Cada uno de estos engranajes nos aporta herramientas, a su vez cada herramienta aprendida y ejecutada nos genera una capacidad de accionar ante situaciones, primero específicas y después generales de nuestra cotidianidad. Pues la paz, a mi entender y por lo que se ha mencionado, son las cosas pequeñas, los actos cotidianos, que nos van alejando de la violencia y nos permiten continuar con el proceso permanente de la construcción de paz.

\section{Paz y pensamiento crítico}

Para hacer o, mejor aún, para construir paz, requerimos ejercitar y poner en marcha con consciencia herramientas y acciones, entre las que podemos mencionar instrumentos psicosociales como la empatía, el respeto, la asertividad y la resiliencia, por mencionar algunas. Pero existe una capacidad — que no es una herramienta como tal—que nos ayuda a entender las herramientas adquiridas y a reflexionar sobre ellas para poder emplearlas en momentos específicos de nuestro día a día con razonamiento y de manera oportuna. Esa capacidad es el pensamiento crítico, que viene de la mano con la construcción de paz y, sobre todo, de la educación para la paz, ya que — gracias a ella y las habilidades que la componen - podemos tomar decisiones sobre problemas, analizar, inferir y tomar una postura ante los conflictos. Esta capacidad nos confronta con nuestras propias valoraciones y con las de otras personas, siempre y cuando se desarrolle de manera adecuada. 
En otros trabajos he mencionado que el pensamiento crítico es un concepto que se ha ido construyendo desde de la Antigua Grecia y que seguimos construyendo hasta nuestros días desde diversos ámbitos del conocimiento humano (Alanis, 2019), por la cual no es fácil de definir. Para no entrar en ambigüedades, a continuación se mencionarán varios autores que se han acercado a explicar o delimitar los márgenes, en ocasiones oscuros, del pensamiento crítico, así como las características que tienen los individuos que lo generan o utilizan.

\section{Pensamiento crítico}

Para comenzar, Robert Ennis (1987) define al pensamiento crítico como la correcta evaluación de enunciados, con tres dimensiones básicas:

a) Dimensión lógica: comprende el acto de juzgar las pretendidas relaciones entre los significados de las palabras y los enunciados.

b) Dimensión criterial: tiene en cuenta el conocimiento de los criterios para juzgar enunciados.

c) Dimensión pragmática: comprende el efecto del propósito latente sobre el juicio y la decisión acerca de si el enunciado es o no suficientemente bueno para lo que se pretende. (Ennis, 1987 citando en Alanis, 2019).

Este autor también menciona que el pensamiento crítico debe centrarse en la toma de decisiones respecto al qué hacer o creer. De igual modo, necesita desarrollarse a partir de analizar enunciados, en la lógica para formalizar el pensamiento. Además, este se puede llevar a la práctica desde emitir juicios de valor hasta resolver, por ejemplo, problemas. Para construir paz, requerimos tomar decisiones de manera asertiva y con conciencia de las consecuencias posibles, es decir, desde la lógica para formalizar nuestro pensamiento. Asimismo, para entender cabalmente los enunciados presentados por otras personas, necesitamos ejercitar la escucha activa, la empatía y el respeto, herramientas que fomentan y alientan la educación para la paz; sin estos elementos, no podemos formalizar el pensamiento y, con ello, los enunciados analizados estarán errados y el ejercicio lógico fallará.

De acuerdo con lo precisado en otro momento (Alanis, 2019, p. 11):

Para McPeck (2016), el pensamiento crítico no viene solo, sino que se tiene que pensar sobre alguna cosa, se tiene que preguntar de manera efectiva sobre problemas concretos existentes, conflictos que estén ahí, puesto que "pensar sobre nada es una imposibilidad conceptual". (Difabio, 2005). A su vez, el conocimiento y la comprensión son los ingredientes principales del pensamiento crítico, esto debido a que el 
conocimiento no es estático, sino reinterpretado y evaluado desde perspectivas diferentes (Tulchin, 1987).

En el caso de las infancias educadas para la paz, se les debe estimular para que apliquen tales habilidades fuera del aula, puesto que enseñar a alguien a ser un pensador crítico implica tanto el dominio cognitivo como el afectivo del aprendizaje de un estudiante en un área (McPeck, 2016). Con ello no se quiere decir que tengamos que reducir los propósitos educativos a desarrollar habilidades mensurables que tienen valor instrumental, puesto que las áreas o disciplinas tienen componentes principales que no deberían ser reemplazadas por habilidades, pero lo que sí hay que hacer es revisar el contenido de la educación.

Por su parte, Harvey Siegel (1990) nos habla de la relación entre pensamiento crítico y racionalidad para que sea un pensamiento a partir de principios, porque los principios implican consistencia, así, el pensamiento crítico es imparcial, consistente y no arbitrario. Por tanto, el pensador crítico piensa y actúa en concordancia con valores, consistencia, justicia e imparcialidad de juicio y de acción ya que conceptualmente se considera como 'crítico' a lo que implica: “juzgar que cumple con los estándares relevantes o criterios de aceptabilidad, y por lo tanto es apropiadamente considerado como "bueno"” (Bailin y Siegel, 2003, citado en Alanis 2019, p. 12). En este sentido, “un pensador crítico no sólo actúa de cierta manera. Un pensador crítico es un cierto tipo de persona” (Siegel, 1990, p. 41).

De la misma forma, quien está educado para la paz, es un cierto tipo de persona, que piensa y acciona bajo estándares diferentes, y que por lo general busca el trabajo en comunidad y la construcción no desde esfuerzos individuales, sino desde la colectividad. Para ello requiere actuar en concordancia con valores, consistencia, justicia, imparcialidad de juicio y de acción.

Muchos de los ideales que se buscan al educar para la paz son presentados por Siegel, quien define criterios para la selección y evaluación de contenidos curriculares, métodos, teorías, políticas y prácticas de enseñanza, en los que resulta indispensable respetar al estudiante como persona, desde una perspectiva del principio kantiano de tratar a los otros como fines y no como medios, así como prepararlos para la vida adulta, introducirlos a la cultura y, en su momento, convertirlos en ciudadanos para que la democracia funcione (Difabio, 2005).

Otro referente para la construcción de un individuo con características particulares - muy especialmente, un alto sentido democrático-es John Dewey y su idea de educación, en la que postula que para generar valores de personalidad humana se requiere de la experiencia del sujeto, pues esta supone un cambio. La experiencia, ya sea en educación formal o fuera de las aulas, nos enfrenta a una reconstrucción continua de sí misma que nos prepara para un futuro remoto, vista como prospectiva y no como retrospectiva. Es así como la experiencia es tanto el punto de partida, como el punto de llegada del aprendizaje (Dewey, 2004). 
La educacion para la paz busca que las acciones tengan una intencionalidad, por ello la experiencia que vamos adquiriendo, tanto de lo que hacemos como de sus consecuencias, nos invita a actuar en funcion de un fin. Así, nuestras experiencias son un impulso y referente, pero tambien se convierten en reflexiones que se cargan de sentido y reoganización de la realidad, una realidad que busca la justicia y la participación informada.

Otro autor que nos habla del pensamiento crítico es Richard Paul, quien considera que este se crea a partir de apropiarse de los contenidos, sobre todo por medio de su internalización y evaluación (Alanis, 2019). En la misma línea, la educación para la paz es el ejercicio pleno de las acciones pacíficas, por lo que no sólo se aprenden teórica o metodológicamente los conceptos y las formas de resolver conflictos, sino que para realizar acciones verdaderamente encaminadas a la construcción de paz desde una cultura de paz, hace falta que se internalicen: creerlo, experimentarlo, pero a la vez entendiendo las repercusiones y consecuencias que las acciones y decisiones conllevan para las otredades y para la propia ipseidad.

Por ello, Paul menciona que a través del pensamiento crítico somos capaces de adquirir conocimiento, comprensión, y habilidades sobre cualquier contenido; para aprenderlo debemos pensar analítica y evaluativamente dentro de ese contenido (Paul y Elder, 2007). Dentro de los límites del construir y ser parte de una sociedad más pacífica, por lo menos desde los parámetros de pensamiento crítico que menciona este autor, no funcionaría si no existe la internalización. Esto podemos verlo con las visiones de nación, de pueblo, de comunidad que cada individuo con poder puede tratar de construir, pues finalmente caerá por su falta de sustento en los individuos que conforman tales sociedades.

Hay que tener en cuenta que al hablar de paz no se trata de un pensamiento sectario o coercitivo, sino de un ejercicio de libertad, puesto que la libertad sólo es posible cuando nos hacemos responsables de nuestros actos y decisiones. Entonces, y solo entonces, llevamos la paz y el pensamiento crítico en un sentido, bajo normas similares, pues son ejercicios disciplinados, autodirigidos, que ejemplifican las perfecciones del pensamiento apropiado a un modo particular de accionar.

Linda Elder y Paul (2007) mencionan que las personas educadas en la paz son capaces de empatizar con otras formas de mirar las cosas, además de poder internalizar conceptos importantes de una disciplina e interrelacionarlos con otros dentro o entre otras disciplinas. Por ello, para que los estudiantes se conviertan en personas educadas, los profesores deben situar el pensamiento en el centro del plan de estudios; deben requerir que los estudiantes trabajen activamente ideas en su pensamiento.

Es Elder la primera en mencionar que para desarrollar pensamiento crítico son necesarias guías, enseñanza y práctica en el monitoreo del pensamiento, usando criterios intelectuales que se puedan aplicar a cualquier disciplina (Elder y Paul, 2003). En este sentido, se requieren herramientas analíticas interdisciplinarias que permitan llegar hasta la lógica más fundamental de cualquier 
disciplina; se necesita aprender a cuestionar propósitos, metas, definiciones de problemas, información, conceptos, etc.

Como ya lo habían mencionado Elder y Paul (2003), el pensamiento crítico es un pensamiento autoguiado y autodisciplinado, que intenta razonar al nivel más alto de calidad. La gente que piensa de manera crítica intenta vivir racional, razonable y empáticamente, además de estar consciente de la naturaleza inherentemente defectuosa del pensamiento humano cuando se deja sin control, esforzándose por disminuir el poder de sus tendencias egocéntricas y sociocéntricas.

Hay que tener en cuenta que, en educación para la paz, muchos de los elementos mencionados están presentes como pilares, bien sea que compartan esta relevancia de manera casual o causal. El punto medular es construir un cierto tipo de individuo a partir de un ejercicio de reflexión, observación y acción en, para y con el mundo. Esto puede resultar, como menciona Elder, al garantizar que los estudiantes aprendan a pensar críticamente y de manera fehaciente, pues nos aseguramos de que los estudiantes no sólo dominen la materia, sino que se conviertan en ciudadanos efectivos, capaces de razonar éticamente y actuar en el bien público (Paul y Elder, 2007).

Finalmente, Facione utiliza el método Delphi ${ }^{3}$ para definir que:

Entendemos que el pensamiento crítico es el juicio auto regulado y con propósito que da como resultado interpretación, análisis, evaluación e inferencia, como también la explicación de las consideraciones de evidencia, conceptuales, metodológicas, criteriológicas o contextuales en las cuales se basa ese juicio. [...] Si bien no es sinónimo de buen pensamiento. Es una combinación entre desarrollar habilidades de pensamiento crítico y nutrir aquellas disposiciones que consistentemente producen introspecciones útiles y que son la base de una sociedad racional y democrática (Asociación Americana de Filosofía, 1990, citado en Facione 2007).

En educación para la paz, se ejercitan y ponen en juego muchas habilidades, capacidades y competencias cognitivas, ante los conflictos o decisiones, las cuales son los fundamentos y la base para entender cómo se compone el pensamiento crítico. Facione (2007) describe, según su consideración, las principales habilidades del pensamiento crítico como:

- Interpretación: comprender y expresar el significado o relevancia de experiencias, situaciones, datos, eventos, juicios, creencias, reglas, procedimientos o criterios.

- Análisis: reconocer las relaciones entre enunciados, preguntas, conceptos, descripciones u otras formas de representaciones para formular creencias, juicios, experiencias, razones, información u opiniones. Esta habilidad

\footnotetext{
${ }^{3}$ Cuyo nombre se inspira en el antiguo oráculo de Delphos. El método Delphi es una técnica de recogida de información que permite obtener la opinión de un grupo de expertos a través de la consulta reiterada.
} 
cuenta con sub-habilidades, las cuales son: examinar ideas, detectar y analizar argumentos.

- Evaluación: primero se lleva a cabo la valoración de la credibilidad de los enunciados, experiencias, situaciones, juicios, creencias u opiniones; después se valora la fuerza lógica de las relaciones de inferencia, reales o supuestas entre enunciados, descripciones, preguntas, etc.

- Inferencia: identificar elementos necesarios para realizar conclusiones razonables, formulación de hipótesis, considerar la información pertinente, y pensar en las consecuencias que se obtienen de los datos, enunciados, principios, evidencias, juicios de valor, creencias, opiniones, conceptos, descripciones, preguntas u otras formas de presentación.

- Explicación: presentar los resultados del razonamiento propio de una manera reflexiva y coherente a alguien más. Esta habilidad cuenta con sub-habilidades: describir métodos y resultados, justificar procedimientos, explicaciones causales y bien razonadas.

- Autorregulación-metacognición: mirar en retrospectiva las actividades cognitivas propias, de los elementos que fueron utilizados en dichas actividades, y de los resultados obtenidos, aplicando particularmente habilidades de análisis y de evaluación de los juicios, con la idea de cuestionar, confirmar, validar, o corregir el razonamiento. Es la cognición sobre la cognición, esto significa tener el conocimiento de nuestro propio conocimiento y actuar sobre él. Aprender a aprender. (Facione, 2007).

\section{Educar en y para la Paz. Construir una Cultura de la paz}

Con el desarrollo de la Irenología ${ }^{4}$, de las diversas fuentes y caminos en que se ha constituido un esquema de conceptos sobre las violencias y las paces, queda claro que no sólo se ha reformulado la concepción de la paz, la guerra y la violencia, sino también constantemente de lo que significa ser humano y de nuestras interacciones con los demás individuos.

Hasta este momento, se ha descrito de la manera más detallada posible las definiciones de educación para la paz y pensamiento crítico, conceptos considerados como indivisibles entre sí. Sin embargo, queda por aclarar un par de puntos que son piedra angular para que este engranaje opere adecuadamente, donde la educación para la paz se transforme, finalmente, en una nueva cultura de paz: eticidad y formación.

En primer lugar, entendemos la eticidad como un proceso de realización de valores en la sociedad y en la propia personalidad, guiado por un interés de

\footnotetext{
${ }^{4}$ Estudios sobre la paz.
} 
emancipación y signado por la socialidad consciente y la moralidad (Camarena, 1995). La realización de valores está estrechamente ligada al accionar, como se venía anunciado al inicio de este artículo, y tiene que ver con un ejercicio práxico; no sólo se trata de entender que existen valores, sino de llevarlos al plano de la acción, donde también requerimos de otredades para llevar a cabo estas acciones de manera cotidiana.

Podemos tener comportamientos propios de nuestro hogar, por supuesto, pero nos enfrentamos a otras conductas, a otras formas de ver el mundo en cuanto cruzamos el umbral de nuestra puerta. Estas contraposiciones son las que generan conflictos que, como se ha visto, pueden ayudar a construir paz, pero debemos tener el respaldo hacia nuestros valores con las herramientas y habilidades que se van ejercitando y potenciando cuando se educa para la paz. En este sentido, no es en sí un ejercicio moral, pues la moral supone algunos principios más rígidos o preestablecidos, sino más bien un ejercicio ético, como explica Sánchez Vázquez (1969):

La ética no crea moral. Aunque es cierto que toda moral efectiva supone ciertos principios, normas o reglas de conducta, no es la ética la que, en una comunidad dada, establece esos principios, o normas. La ética se encuentra con una experiencia histórico-social en el terreno de la moral, o sea, con una serie de morales efectivas ya dadas, y partiendo de ellas trata de establecer la esencia de la moral, su origen, las condiciones objetivas y subjetivas del acto moral, las fuentes de la valoración moral, la naturaleza y función de los juicios morales, los criterios de justificación de dichos juicios, y el principio que rige el cambio y sucesión de diferentes sistemas morales. (p. 21-22)

Por lo anterior, los ejercicios reflexivos acerca de las estructuras dadas se convierten en los principales catalizadores para que la moral no se vuelva estática, rígida e inflexible, sino por el contrario, para que llevemos a cabo este proceso de eticidad entendiendo los resultados de tales reflexiones como un producto que se va reformando y reconfigurando a cada paso, pues el ejercicio reflexivo va dando formas nuevas de convivencia que se han de poner sobre la mesa para discutir su pertinencia y cómo repercuten en el futuro de la comunidad, sobre todo en su carácter emancipatorio.

Como proceso, lo anterior requiere su tiempo y exige esfuerzos de cada uno de los individuos de una comunidad; a su vez, envuelve a cada comunidad en su conjunto y adquiere los matices de esta, mientras se va relacionando con otras comunidades, que finalmente están constituidas por individuos que transitan entre comunidades, organizaciones e instituciones y van generando y construyendo estos procesos. Pero este proceso no se da de manera espontánea o por mera voluntad de los individuos.

Por otro lado, en segundo lugar, tenemos la formación. Esta se refiere al proceso por el que el sujeto se constituye como tal, a partir de sus objetivaciones (Camarena, 1995). Ya sea en instituciones educativas o fuera de ellas, el sujeto 
está permeado por las relaciones con otredades; ahí el sujeto se forma al crear cultura, pues en ese proceso transforma la realidad y se trasforma a sí mismo. No trataré siquiera de definir lo que es cultura, pues se torna una tarea imposible, inabarcable e inacabable, sin embargo, cuando se habla de cultura de paz, tenemos límites un poco menos escabrosos, un tanto más delimitados en cuanto una visión y acción de las relaciones, un ejercicio práxico ante el mundo y una ética y moral de ciertas características, constatando que no se trata de una utopía, sino de una construcción gradual, de un proceso continuo y de permanente transformación.

Ciertamente, la formación también implica la adquisición de competencias para plantearse problemas - y no sólo para resolverlos bajo ciertos esquemas aprendidos y repetitivos-, para enfrentar situaciones inéditas en todos los ámbitos posibles y para participar de manera intencional, reflexiva, crítica y creativa en la preservación, generación y transformación de la cultura, así como en el desarrollo, crítica y transformación de los órdenes normativos sociales. Por tanto, la formación, en conjunción con la eticidad, se convierte en el punto de soporte para construir una cultura de paz desde la transformación de conflictos y la reflexión y crítica a las estructuras que podamos observar violentas.

Para lograr el cambio, la formación incluye y supera tres procesos que nos menciona Camarena (1995), a saber:

- Cultivo: Proceso que contribuye al desarrollo de la personalidad. Aquí se adquieren competencias y se asegura la identidad, interactuando con el medio natural y con otros, la interacción no implica cercanía.

- Enculturación: La apropiación de la cultura. Aquí adquirimos todos los conocimientos y los objetos reales o simbólicos en los que estos saberes se concretan.

- Socialización: La incorporación del particular a ciertas integraciones sociales, gracias a que ha internalizado ciertos órdenes normativos considerados legítimos. El individuo no sólo interactúa con otros, sino que estos se influyen mutuamente mediante reglas tácitas o enunciadas para cohabitar o afiliarse en grupos más cercanos que comparten ciertas características y con ello afianzarse como individuos.

Este proceso se lleva a cabo cotidianamente, por ello es indispensable que se haga de manera consciente y reflexiva, especialmente en la formación académica, pues en el ámbito escolar estos tres están presentes y se inducen de manera deliberada y sistemática, propiciando al educando medios y herramientas para que adquiera no sólo aprendizajes académicos, sino los necesarios para desarrollarse, socializar y apropiarse de su cultura.

Podemos ver este proceso como un estado dinámico de acciones, como menciona Hernández (2021), pues convergen de manera interactiva las dimensiones personal, social, cognitiva, motivacional, ética y emocional. Al ser capacidades, 
tienen la característica que pueden aprenderse, por lo que resulta pertinente integrarlas en todos los procesos formativos y organizativos.

\section{Conclusiones}

La paz se ha entendido no sólo como la ausencia de guerra, sino como un concepto mucho más complejo que incluye, pero no se limita, a la justicia, al desarrollo económico y social; así como el desarrollo holístico de los individuos, desde lo cognitivo, social, emocional, hasta el desarrollo de sus capacidades y habilidades. A su vez, la paz también tiene que ver con la forma en que transformamos los conflictos, en la que nos enfrentamos al mundo, con nuestras propias habilidades y capacidades. De igual manera, la paz engloba nuestra capacidad y pensamiento crítico, ya que al estar formados de diversas habilidades podemos llevar a cabo ejercicios de diferente índole para ir construyendo nuestra realidad.

En cuanto a la construcción de la realidad, también se menciona la influencia de la cultura en los individuos, que a su vez pueden permear esta misma cultura para transformarla, una vez más, con un proceso consciente y emancipador. Por lo que no basta con hablar de paz por sí misma, hay que modificar la cultura preeminentemente violenta, y este proceso sólo se logra a través de la educación. Una educación que fomente una personalidad, una apropiación de la cultura y una integración social desde la reflexión, pues sin ella los patrones de conducta, actitudes, prejuicios y dogmas violentos simplemente se perpetúan y solidifican, como hasta hoy.

La construcción de paz no es una tarea sencilla ni un camino fácil de recorrer. Desde su misma concepción, nos confronta con nuestras propias valoraciones, prejuicios y dogmas; nos pone en situaciones difíciles, donde debemos tomar la decisión de cambiar lo que hacemos y cómo lo hacemos; enfrentarnos a la incertidumbre o perpetuar lo que es sabido y lo violento, obteniendo resultados ya conocidos, pero que en poco o nada aportan al mejoramiento de nuestra condición humana. La pregunta real sería si estamos dispuestos a ser responsables y conscientes de nuestras palabras, de nuestras acciones y de las consecuencias de una manera reflexiva y consciente. 


\section{Referencias}

Alanis, J. (2015). Intervención filosófica en el curso-taller "equidad y filosofía" para $5^{\circ}$ y $6^{\circ}$ de primaria. (Tesis de Licenciatura). Universidad Nacional Autónoma de México, México. Recuperado de https://repositorio.unam.mx/ contenidos/303667.

Alanis, J. (2019). Desarrollo de habilidades de pensamiento crítico a través de un programa de filosofía para niños. (Tesis de maestría). Universidad Autónoma del Estado de Morelos. Recuperado de http://riaa.uaem.mx/xmlui/handle /20.500.12055/686.

Bailin, S. y Siegel, H. (2003). Critical Thinking. En N. Blake, P. Smeyers, R. Smith y P. Standish, The Blackwell Guide to the Philosophy of Education. Main: Blackwell. pp. 181-193.

Bosé, M., y Fernández, D. (2017). La historia de la paz en Occidente. University for Peace.

Camarena, M. (1995). Eticidad, valores sociales y educación. Universidad Pedagógica Nacional.

Cascón, F. (2001). Educar en y para el conflicto. Bellaterra: Cátedra UNESCO sobre Paz y Derechos Humanos, Universidad Autónoma de Barcelona.

De Vera, F. (2016). La construcción del concepto de paz: paz negativa, paz positiva y paz imperfecta. Cuadernos de estrategia, (183), pp. 119-146.

Descartes, R. (2011). René Descartes I. Madrid: Editorial Gredos.

Dewey, J. (2004). Democracia y educación. Una introducción a la filosofía de la educación. Madrid: Ediciones Morata.

Difabio, H. (2005). El "critical thinking movement" y la educación intelectual. Estudios sobre educación. Estudios sobre educación, 9, pp. 167-187. Recuperado de dadun.unav.edu/bitstream/10171/8919/1/NE.PDF.

Elder, L. y Paul, R. (2003). Los fundamentos del Pensamiento analítico. Recuperado de http://www.criticalthinking.org/resources/PDF/SP-Pensamientoa nal\%C3\%ADtico.pdf

Ennis, R. (1987). Critical thinking and the curriculum. En M. Heiman y J. Slomianko (eds.), Thinking Skills Instruction: Concepts and Techniques. Building Students' Thinking, pp. 40-48. Washington: National Education Association. Recuperado de https://files.eric.ed.gov/fulltext/ED306559.pdf.

Facione, P. (2007). Pensamiento Crítico: ¿Qué es y por qué es importante? California: Insight Assessment. Recuperado de https://eduteka.icesi.edu.co/ pdfdir/ PensamientoCriticoFacione.pdf.

Galtung, J. (2003). Tras la violencia 3R: reconstrucción, reconciliación, resolución: Afrontando los efectos visibles e invisibles de la guerra y la violencia. Euskadi: Bakeaz.

Hernández, E. M. L., Villaseñor, E. L., y Díaz, A. G. (2021). Opiniones de estudiantes universitarios asociadas a capacidades resilientes desarrolladas durante la pandemia Covid-19. Una visión desde las dimensiones emocional, 
ética y cognitiva. DEDiCA Revista de Educação e Humanidades, 18, pp. 213-234.

Islas, V. C. (2019). Percepciones de los adolescentes sobre la violencia, guía de trabajo hacia la construcción de paz. (Tesis de maestría). Universidad Autónoma del Estado de Morelos. Recuperado de http://riaa.uaem.mx/ xmlui/handle/20.500.12055/517

Jares, X. (1999). Educación para la paz. Su teoría y su práctica. Madrid: Popular. Jares, X. (2001). Educar para la paz y la convivencia: tarea de todas y todos. Recuperado de https://studylib.es/doc/8412645/educar-para-la-paz-y-la-con vivencia--tarea-de-todas-y.

Jiménez, F. (2011) Racionalidad pacífica. Una introducción a los Estudios para la paz. Madrid: Dykinson.

Kant, E. (2014). Crítica de la Razón Pura. Barcelona: Gredos.

Martín J. (2003). Los sentidos de la violencia. Granada: Universidad de Granada.

McPeck, J. (2016). Critical thinking and education, precise its suggested solutions. Londres: Routledge.

Muñoz, A. (2001). La paz imperfecta ante un universo en conflicto. La paz imperfecta. Granada: Universidad de Granada.

Paul, R. y Elder, L. (2007). Critical Thinking Competency Standards. Journal of Developmental Education, 35, (3). Recuperado de https://files.eric.ed.gov/ fulltext/EJ998804.pdf.

Platón. (2014). Diálogos II. Madrid: Editorial Gredos.

Platón. (2017). Diálogos I. Madrid: Editorial Gredos.

Sánchez, A. (1969). Ética, Ciudad de México: Grijalbo.

Siegel, H. (1990). Educating reason. Rationality, critical thinking and education. Nueva York: Routledge.

Smith, D. (1979). Estudio de los conflictos y educación para la paz. Perspectivas. Revista Trimestral de Educación, IX, (2).

Tulchin, J. B. (1987). Más allá de los hechos históricos: sobre la enseñanza del pensamiento crítico. Revista de educación, 282, pp. 235-253.

Organización Mundial de la Salud. (2002). Informe mundial sobre la Violencia y la Salud. Washington: Organización Panamericana de la Salud. 